\title{
Evaluation of the influence of sensor geometry and physical parameters on impedance-based structural health monitoring
}

\author{
L.V. Palomino ${ }^{\mathrm{a}, *}$, K.M. Tsuruta ${ }^{\mathrm{a}}$, J.R.V. Moura, Jr. ${ }^{\mathrm{a}}$, D.A. Rade ${ }^{\mathrm{a}}$, V. Steffen, Jr. ${ }^{\mathrm{a}}$ and D.J. Inman ${ }^{\mathrm{b}}$ \\ ${ }^{a} F e d e r a l$ University of Uberlandia, Campus Santa Monica, Uberlandia-MG, Brazil \\ ${ }^{\mathrm{b}}$ Department of Aerospace Engineering, University of Michigan, MI, USA
}

\begin{abstract}
Structural Health Monitoring (SHM) is the process of damage identification in mechanical structures that encompasses four main phases: damage detection, damage localization, damage extent evaluation and prognosis of residual life. Among various existing SHM techniques, the one based on electromechanical impedance measurements has been considered as one of the most effective, especially in the identification of incipient damage. This method measures the variation of the electromechanical impedance of the structure as caused by the presence of damage by using piezoelectric transducers bonded on the surface of the structure (or embedded into it). The most commonly used smart material in the context of the present contribution is the lead zirconate titanate (PZT). Through these piezoceramic sensor-actuators, the electromechanical impedance, which is directly related to the mechanical impedance of the structure, is obtained as a frequency domain dynamic response. Based on the variation of the impedance signals, the presence of damage can be detected. A particular damage metric can be used to quantify the damage. For the success of the monitoring procedure, the measurement system should be robust enough with respect to environmental influences from different sources, in such a way that correct and reliable decisions can be made based on the measurements. The environmental influences become more critical under certain circumstances, especially in aerospace applications, in which extreme conditions are frequently encountered. In this paper, the influence of electromagnetic radiation, temperature and pressure variations, and ionic environment have been examined in laboratory. In this context, the major concern is to determine if the impedance responses are affected by these influences. In addition, the sensitivity of the method with respect to the shape of the PZT patches is evaluated. Conclusions are drawn regarding the monitoring efficiency, stability and precision.
\end{abstract}

Keywords: Structural health monitoring, damage detection, piezoceramic sensor-actuators

\section{Introduction}

SHM techniques have been lately receiving great interest from the academic and industry communities that are interested in aerospace and civil structures, vehicles and machines [1]. Among the SHM techniques, the method based on the electromechanical impedance is considered to be one of the most promising [2]. It is a nondestructive method that explores the electro-mechanical coupling property of piezoelectric materials to monitor the occurrence and evolution of structural damage. The application of this technique consists in bonding piezoelectric materials, more frequently in the form of thin patches, to the monitored structure. The electromechanical behavior of this system can be characterized by the electromechanical-impedance, which is frequency-dependent, defined as the ratio between the input voltage and the electrical current. This function depends also on the mechanical features (inertia and stiffness of the base structure). Thus, through the variations observed in the electromechanical impedance responses, it is possible to assess information about structural changes induced by damage. This possibility is related to the electrical impedance of the PZT patch being directly associated with the mechanical impedance of

\footnotetext{
*Corresponding author: L.V. Palomino, Federal University of Uberlandia, Campus Santa Monica, Av. João Naves de Avila N. 2121, Bloco 1M, Uberlandia-MG, Brazil. Tel.: +55 3432394147; Fax: +55 3432394206; E-mail: lvpalomino@ mecainca.ufu.br.
} 
structure to which it is bonded. By using the same piezoelectric element as both sensor and actuator, a small number of components and cables are necessary [2]. For the monitoring success of this technique, the system must be robust to environmental variations from different sources [3,4]. Otherwise, environmental changes could influence the measurements, undermining the reliability of the procedure. Moreover, the process of monitoring the structural integrity can identify a situation of false positive during the evaluation of a given structures. Consequently, the evaluation of the effects caused by sensor geometry and physical parameters and the possibility of occurrence of false diagnostics is highly recommended. This work presents a preliminary study of the influence of the shape of the PZT patch on the impedance measurement; moreover the influences of electromagnetic radiation, temperature and pressure, and ionic environment on the impedance-based structural health monitoring method are analyzed.

\subsection{Impedance-based structural health monitoring}

The technique known as impedance-based structural health monitoring uses the piezoelectric properties of the PZT patch that is installed in the structure being tested and is considered as a non-destructive damage evaluation method [2]. The basic idea behind this technique is to monitor the changes in the structure's mechanical impedance caused by the presence of damage. Since the direct measurement of the mechanical impedance of the structure is a difficult task, the method uses piezoelectric materials bonded to or incorporated into the structure, allowing the measurement of the electrical impedance. This measurement is related to the mechanical impedance of the structure, which is affected by the presence of damage. Evidently, it is considered that the piezoelectric sensor-actuator used in the monitoring procedure remains intact along the test.

The impedance-based SHM technique was first proposed by Liang et al. [5] and subsequently the method was extended by Chaudhry et al. [6,7], Sun et al. [8], Park et al. [2,9], Soh et al. [10], Bhalla et al. [11] Giurgiutiu et al. [12] Moura and Steffen [4], Peairs [13], Moura [14], Liu et al. [15], Neto et al. [16] and Zhang et al. [17].

The impedance-based monitoring method uses simultaneously both versions, direct and inverse, of the piezoelectric effect [2]. When the PZT patch is bonded to the structure and a low electric voltage is applied, generally $1 \mathrm{~V}$ [18], a strain is produced in the PZT patch. Using a high frequency of excitation (as compared to typical modal analysis testing), the dynamic response of the structure represents only the local area of the sensor and is not affected by the boundary conditions. Then, the response of the mechanical vibrations is transmitted to the sensor in the form of an electrical response. When an incipient damage leads to changes in the dynamic response (given by the impedance signal), this is observed in the electric response of the PZT patch. The electromechanical model that quantifies and describes the measurement process is illustrated in Fig. 1 for a single-degree-of-freedom system.

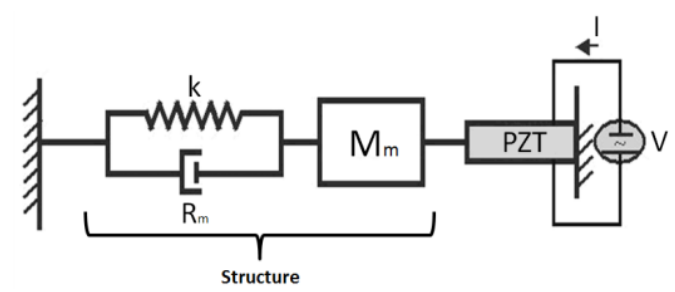

Fig. 1. Model used to represent a single-degree-of-freedom structure containing a PZT patch.

For this system, Liang et al. [5] demonstrated that the admittance $(Y(\omega)$ inverse of the impedance) of the PZT patch can be written as a function of the combined PZT actuator and structure mechanical impedance, as given by Eq. (1):

$$
Y(\omega)=i \omega a\left(\bar{\varepsilon}_{33}^{T}(1-i \delta)-\frac{Z_{s}(\omega)}{Z_{s}(\omega)+Z_{a}(\omega)} d_{3 x}{ }^{2} \hat{Y}_{x x}^{E}\right)
$$

where $Z_{a}(\omega)$ and $Z_{s}(\omega)$ are the PZT material's and the structure's mechanical impedances, respectively. $\hat{Y}_{x x}^{E}$ is the complex Young's modulus of the PZT with zero electric field, $d_{3 x}$ is the piezoelectric coupling constant in the arbitrary x direction at zero electric field, $\varepsilon_{33}^{T}$ is the dielectric constant at zero stress, $\delta$ is the dielectric loss tangent of 
the PZT, and $a$ is a geometric constant of the PZT. Assuming that the mechanical properties of the PZT patch do not vary over the time used for monitoring, Eq. (1) shows that the electrical impedance of the PZT patch is directly related to the structure's impedance. Damage causes changes in the structure's mechanical impedance, thus changing local dynamical features. Hence, the electrical impedance is used to monitor the health of the structure as represented by the mechanical impedance of the structure.

It is necessary for the excitation wavelength to be smaller than the characteristic length of the damage to be detected. According to the literature [2], the frequency range typically used in the impedance-based method is from $30 \mathrm{kHz}$ to $250 \mathrm{kHz}$. The effective range for a given structure is determined by trial and error or by optimization procedures [14].

As for the sensitive region to identify changes, Park et al. [2] claim that for a simple PZT a damage located at a distance (radial) of up to $0.4 \mathrm{~m}$ can be identified in composite materials and up to $2 \mathrm{~m}$ in bars consisting of a single metal.

The curve that represents the impedance response provides a qualitative assessment of the damage influence. For a quantitative assessment of the failure, a previously defined damage metric is used [19]. In the present contribution the mean absolute percentage deviation metric (MAPD) was used. The corresponding mathematical formula for MAPD is given by Eq. (2):

$$
M A P D=\sum_{i=1}^{n}\left|\frac{\left(\operatorname{Re}\left(Z_{1, i}\right)-\operatorname{Re}\left(Z_{2, i}\right)\right)}{\operatorname{Re}\left(Z_{1, i}\right)}\right|
$$

where $Z_{1, i}$ is the impedance of the PZT measured at healthy conditions (baseline) and $Z_{2, i}$ is the impedance for the comparison with the baseline measurement at the frequency interval $i$.

Reliable theoretical models are not available in many SHM applications. The application of statistics techniques, such as the factorial design has been used in various engineering problems, as reported by Box and Draper [20] and Myers and Montgomery [21]. Consequently, it is possible to use models that have been previously numerically adjusted to represent the phenomenon studied. An interesting alternative uses meta-models, which are built from experimental tests. The literature presents different meta-model techniques and the one that has been chosen for the present contribution is the so-called RSM (Response Surface Methodology). This approach encompasses a set of statistical techniques aiming at obtaining representative meta-models that are able to provide information regarding the behavior of the system for a delimited design space. Typically, analyses of experiments are used to relate the responses to quantitative factor levels, so that the behavior of the system can be studied. As the mathematical law that relates the responses to the factors is often unknown, a low order polynomial is commonly used to represent the relation between the response and the explanatory variables (factors). In this work, the meta-model results are presented by using two plot graphics, namely the main effects plot and the interaction plot. The main effects plot shows the average outcome for each value of each variable, combining the effects of the other variables as if all variables were independent (main effects refer to the primary factors of interest in the experiment), and the interaction plots illustrate the effects between variables which are not independent (cross effects).

\section{Sensor geometry}

As stated above, the impedance-based structural health monitoring uses piezoelectric patches coupled to the structure. The impedance signals obtained should be sensitive to physical changes in the structure in order to detect incipient damage. The study of the shape of the piezoelectric patch aims at improving the efficiency of the technique application. Two shapes of piezoelectric patches, namely circular and square have been tested in the laboratory. They were bonded to two different types of structures, namely a plate and a beam.

Two square and two circular PZT patches with the same size were bonded to each side of the specimens as presented in Fig. 2a, b. A mass of $3.0 \mathrm{~g}$ was used to represent damage (Fig. 2c). The impedance measurements were acquired before and after the mass has been added, at the position A, for each specimen. To record the data, an HP4194A impedance analyzer was used and 16 averages were taken for the collected data. The frequency bands observed were from $60 \mathrm{kHz}$ to $70 \mathrm{kHz}$ for the plates and from $80 \mathrm{kHz}$ to $88 \mathrm{kHz}$ for the beams, with $20 \mathrm{~Hz}$ of resolution. 


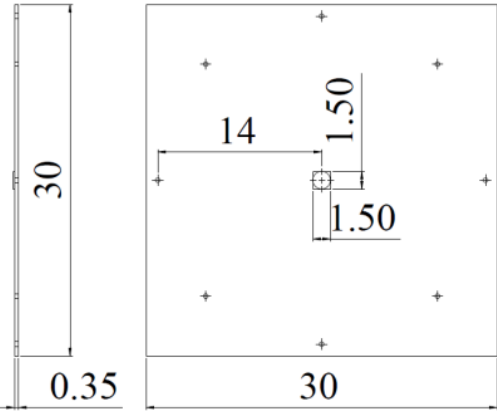

(a)

SIDE A

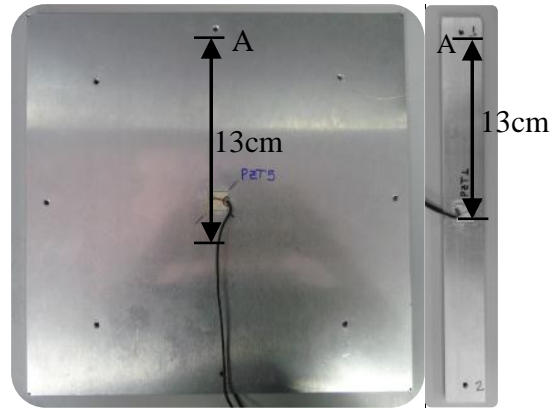

(b)

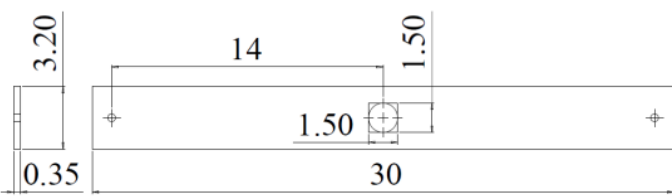

SIDE B

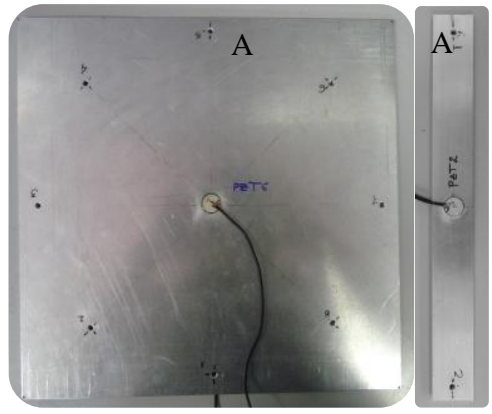

(c)
Damage

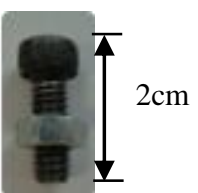

(d)

Fig. 2. (a) Geometry of the plate and beam; (b) Side A of the plate and beam; (c) Side B of the plate and beam; (d) Mass added to the structure.

The impedance signals of the plate and beam can be observed in Fig. 3 for various configurations. As the impedance signal changes clearly for each configuration considered it can be concluded that the impedance-based technique was sensitive enough to detect the inserted damage in the structures.

The damage metric values are now used to build a meta-model for the verification of the effect of the shape of the PZT patch (Eq. (3) - for the beams; Eq. (4) - for the plates):

$$
\begin{aligned}
& D M=51.8635-35.9613 \text { State }+0.9213 \text { Shape } \\
& R-S q=98.09 \% \quad R-S q(\text { adj })=98.01 \% \\
& D M=16.4709-9.0879 \text { State }+0.2503 \text { Shape } \\
& R-S q=96.43 \% \quad R-S q(\text { adj })=96.27 \%
\end{aligned}
$$

The results presented in Fig. 4 show that the greatest contribution of an isolated effect is the one due to the added mass (as compared with the influence of the shape of the PZT patch).

\section{Electromagnetic radiation}

The methodology adopted in this study consists in testing an aluminium beam under the influence of an electromagnetic field, Fig. 5a. The damage was simulated by adding a mass $(1 \mathrm{~g}$ or $2 \mathrm{~g})$ to the beam at the circular mark on the structure as shown in Fig. 5b. The specimen was tested under a magnetic field, and measurements of electromechanical impedance were made before and after applying the magnetic field and adding a mass to the system. 


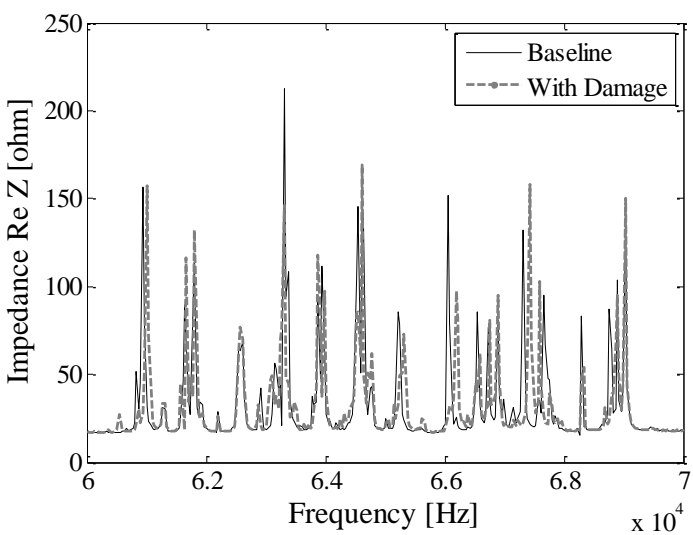

(a)

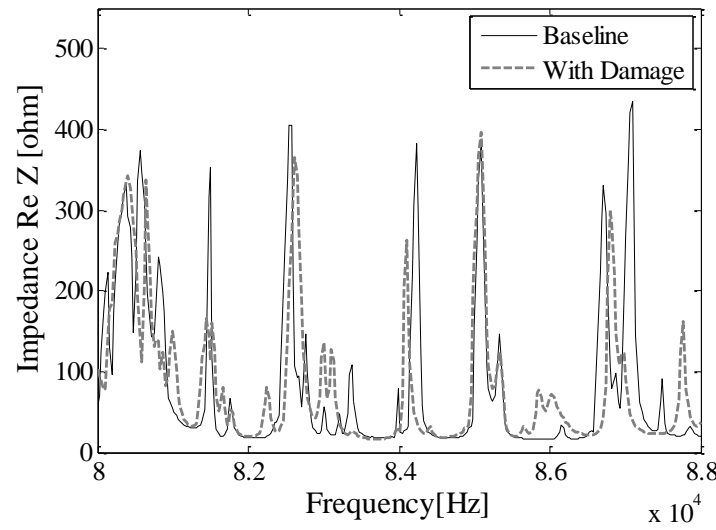

(c)

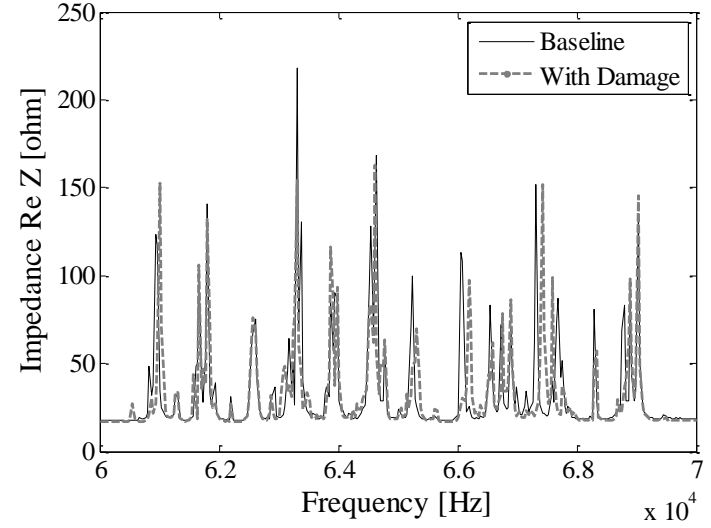

(b)

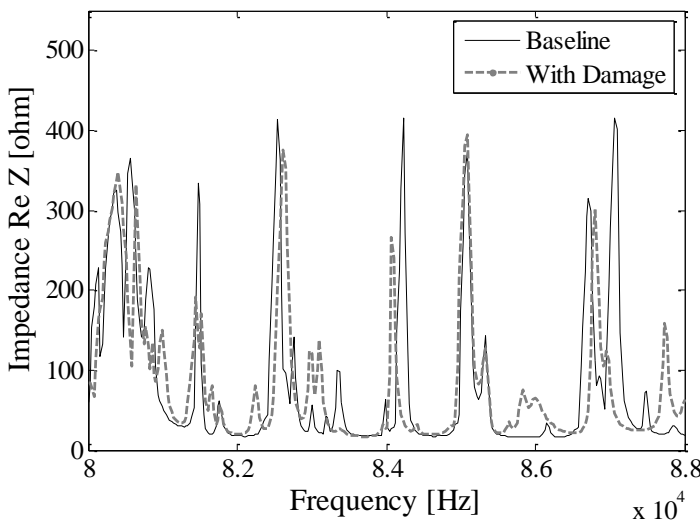

(d)

Fig. 3. Impedance measurement: (a) Square PZT patch on the plate; (b) Circular PZT patch on the plate; (c) Square PZT patch on the beam; (d) Circular PZT patch on the beam.

For the generation of the electromagnetic field two different voltages were applied in the copper coil: $2 \mathrm{~V}$ and $4 \mathrm{~V}$. According to the Eq. (5), obtained from the Biot-Savart and Ampere laws, the values of the generated field can be easily computed.

$$
B=\frac{\mu_{0} N I_{c}}{2 R}
$$

where $\mu_{0}=1.2566 \times 10^{-6} \mathrm{Tm} / \mathrm{A}$ (magnetic constant), $N=18$ turns, $I_{c}=$ Voltage $/ 0,5 \Omega, R=0.04 \mathrm{~m}$ (average radius of the turns). Thus, from Eq. (5), the electromagnetic fields $B$ are calculated as $1.13094 \times 10^{-3} \mathrm{~T}$ and $2.26188 \times 10^{-3} \mathrm{~T}$ for 2 and $4 \mathrm{~V}$, respectively.

To record the data, an HP4194A impedance analyzer was used and 16 averages were taken for the collected data. The frequency band observed was from $24 \mathrm{kHz}$ to $34 \mathrm{kHz}$, with $20 \mathrm{~Hz}$ of resolution.

The impedance measurements obtained for the PZT patch are shown in the Fig. 6. The first plot is a comparison between the data obtained without the addition of mass for different electromagnetic fields. The other figures show a comparison of the baseline with the case in which mass was added to the beam for each voltage considered. The first plot shows that there is no difference in the signals obtained.

The damage metric values are now used to build a meta-model for the verification of the effect of the electromagnetic field. The meta-model is given by Eq. (6): 


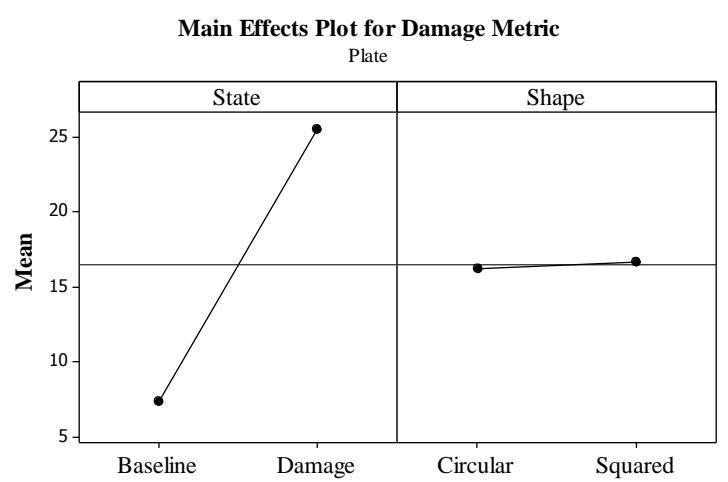

(a)

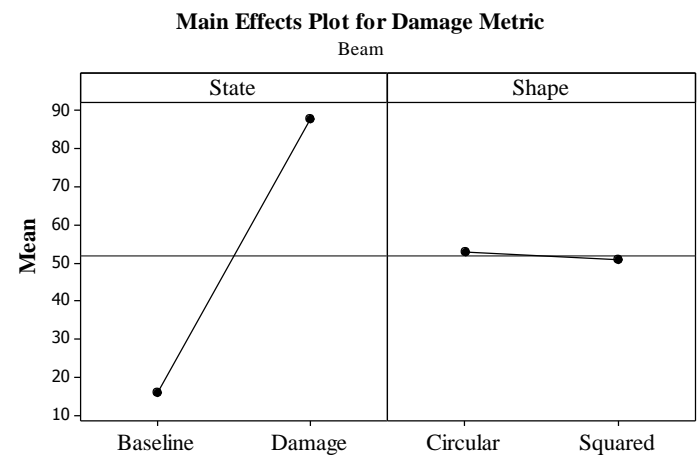

(c)

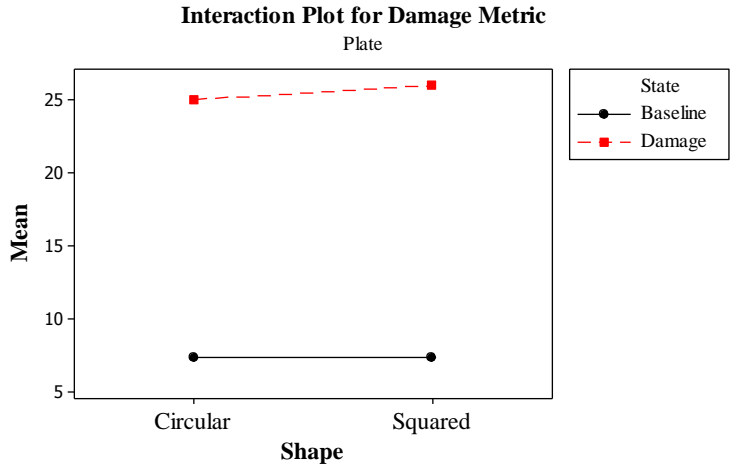

(b)

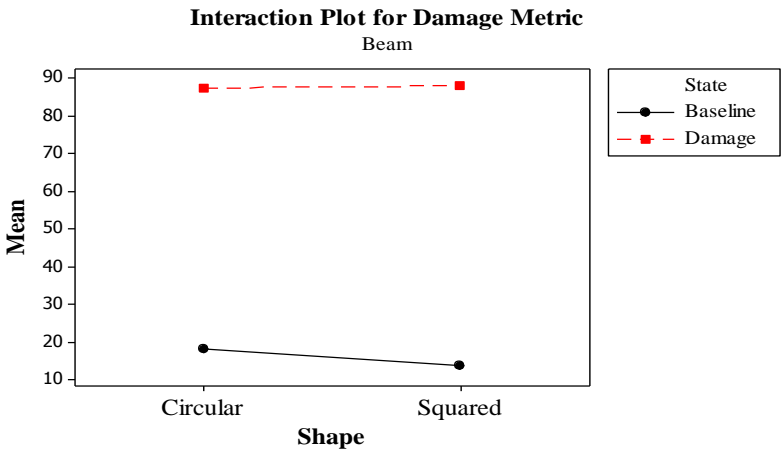

(d)

Fig. 4. Main effects of the sensor shape (a) Isolated effects for the plate; (b) Cross effects for the plate; (c) Isolated effects for the beam; (d) Cross effects for the beam.

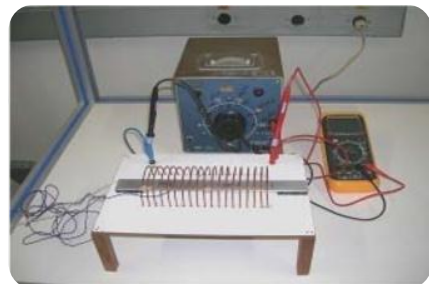

(a)

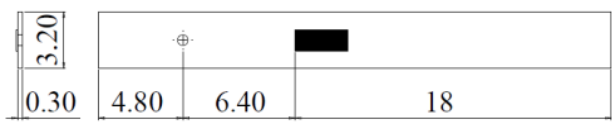

(b)

Fig. 5. (a) Experimental arrangement; (b) Geometry of the specimen and position of the PZT patch.

$$
\begin{aligned}
& D M=1.2896+72.5019 m+0.7737 V-13.8849 m^{2}-0.0228 V^{2}-0.5582 m V \\
& R-S q=100.0 \% \quad R-S q(\text { adj })=100.0 \%
\end{aligned}
$$

where $m$ is the adding mass, $V$ is the voltage applied to generate the magnetic field and $D M$ is the damage metric.

The results presented in Fig. 7 show that the greatest contribution of an isolated effect is the one due to the mass (as compared with the influence of the electric field). Observing the figure showing the cross effects, Fig. 7b, it is possible to notice that the voltage change leads to a slight inclination, however the change of the mass leads to a significant variation of the plots. 


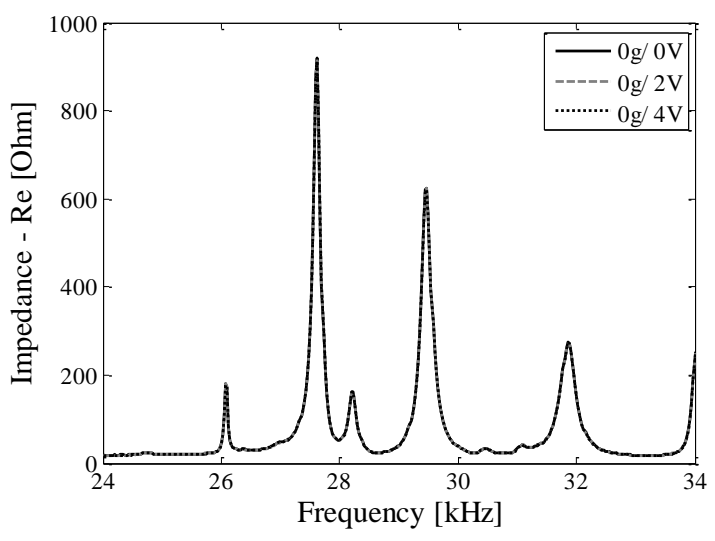

(a)

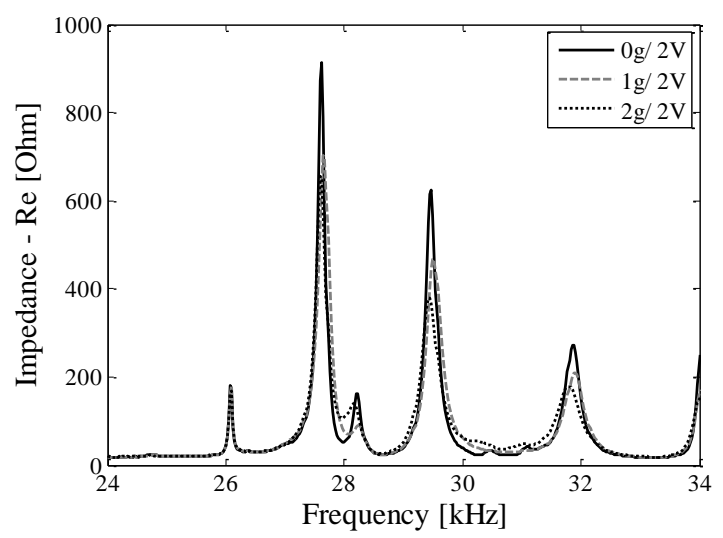

(c)

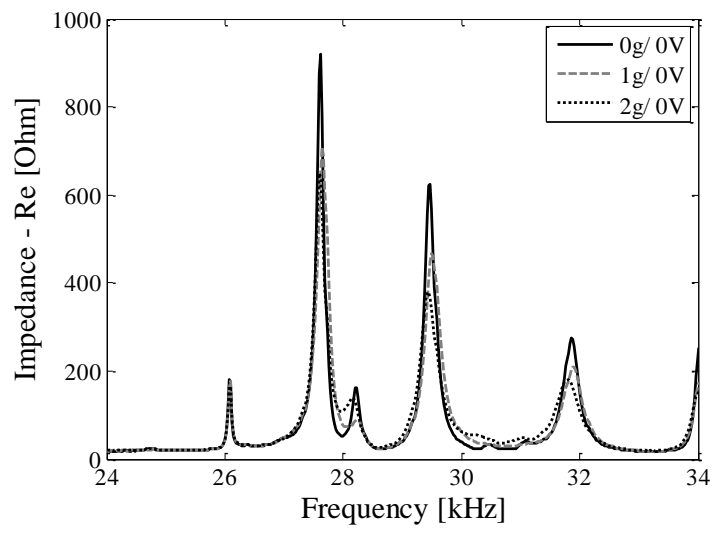

(b)

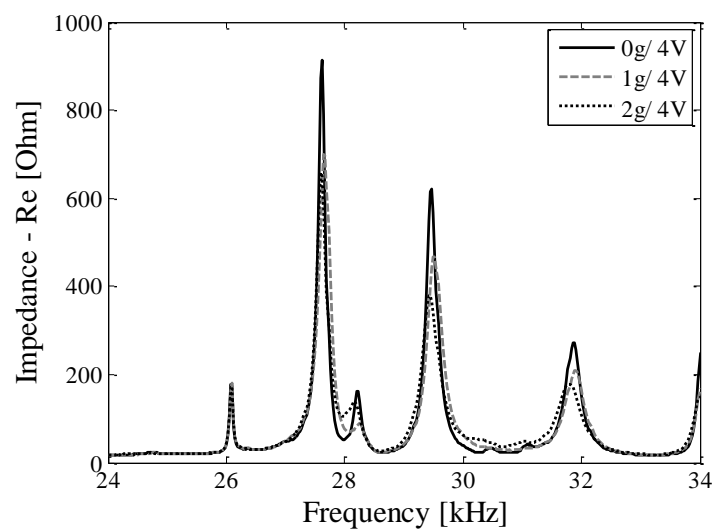

(d)

Fig. 6. Impedance Measurement: (a) varying the electromagnetic field $(0 \mathrm{~V}, 2 \mathrm{~V}$ and $4 \mathrm{~V})$; (b) varying the added mass to the structure for constant electromagnetic field $(0 \mathrm{~V}) ;(\mathrm{c})$ varying the added mass to the structure for constant electromagnetic field $(2 \mathrm{~V})$; (d) varying the added mass to the structure for constant electromagnetic field $(4 \mathrm{~V})$.

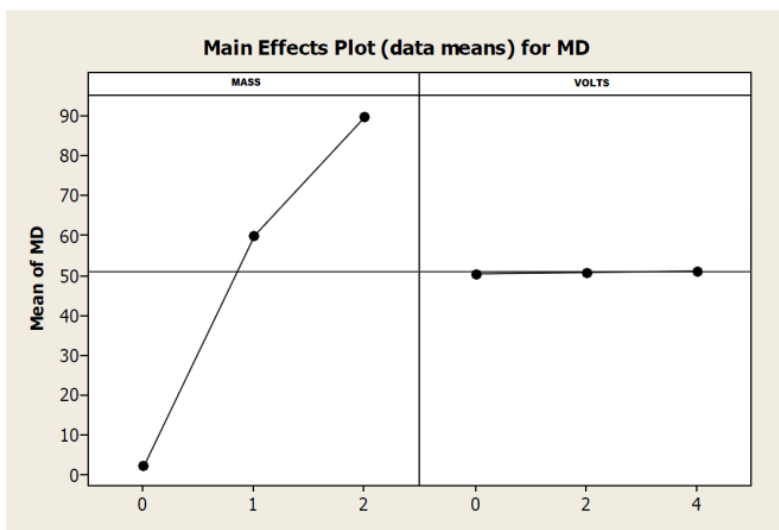

(a)

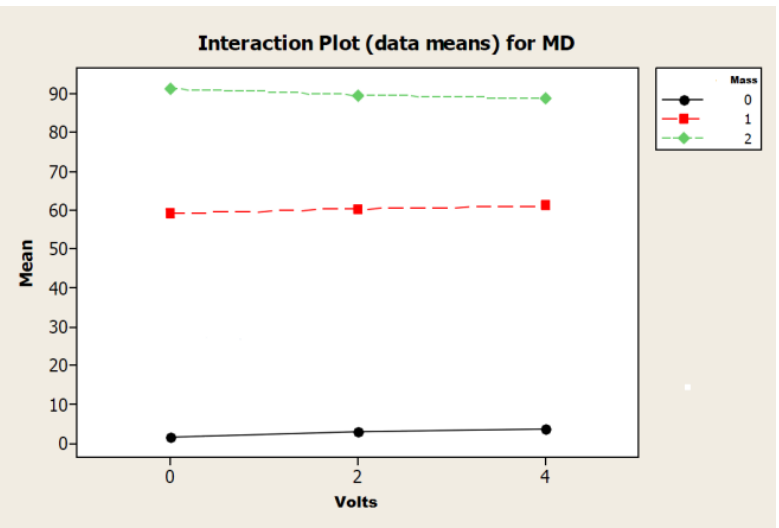

(b)

Fig. 7. Main effects of electromagnetic field: (a) Isolated effects; (b) Cross effects. 


\section{Ionic environment}

The structure used was again an aluminium beam of approximately $40 \mathrm{~cm}$ in length (Fig. 8a). The damage was represented by a mass ( $2.3 \mathrm{~g}$ or $8.5 \mathrm{~g}$ ) that was added to the beam at the position represented by the circular mark shown in the Fig. 8b. During the tests, the aluminium beam was submerged in a container with the following conditions: only water, water with $29.2 \mathrm{~g}$ of aquarium salt (50\% salt) and water with $58.3 \mathrm{~g}$ of aquarium salt (full aquarium condition, $100 \%$ salt). All tests were made with the same amount of water, $1750 \mathrm{ml}(1.75 \mathrm{~L})$ in a plastic reservoir.

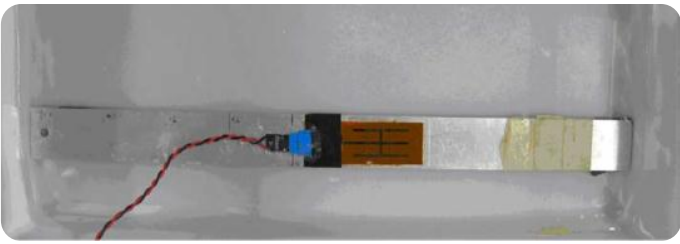

(a)

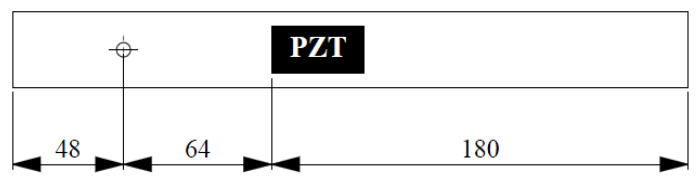

(b)

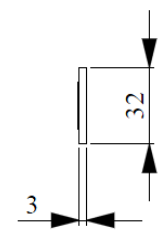

Fig. 8. (a) Experimental assembly; (b) Geometry of the specimen and position of the PZT patch.

To record the data, an HP4194A impedance analyzer was used and 16 averages were taken for the collected data. The frequency band observed was from $76 \mathrm{kHz}$ to $85 \mathrm{kHz}$, with $20 \mathrm{~Hz}$ of resolution.

The impedance measurements obtained for the PZT patch are shown in the Fig. 9. A comparison involving the data obtained for the case without the addition of mass and the impedance data for the various values of mass was made. It can be concluded that the ionic environment has a great influence in the impedance measurements.

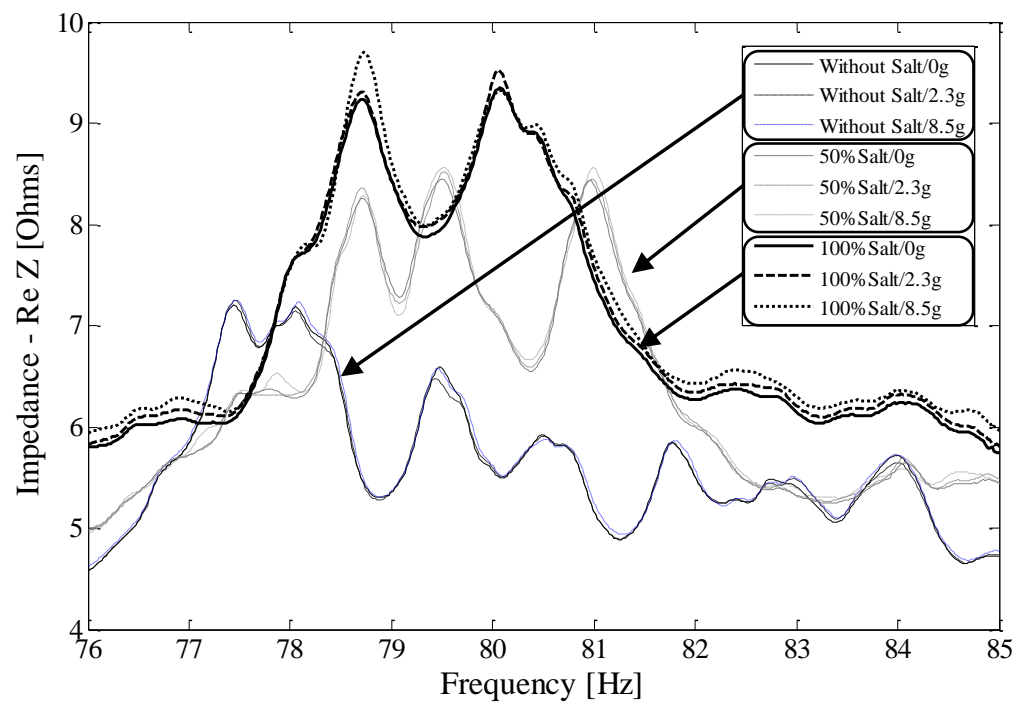

Fig. 9. Impedance measurements - Ionic Environment.

A full factorial design was performed by considering two factors, namely the mass (damage) and the ionic influence (aquarium salt). The purpose of this procedure is to analyze the influence of each factor in the impedance responses. For this aim a meta-model was constructed as given by Eq. (7):

$$
\begin{aligned}
& D M=3.14+53.7 \text { salt }+2.27 \text { mass } \\
& R-S q=98.3 \% \quad R-S q(\text { adj })=97.8 \%
\end{aligned}
$$




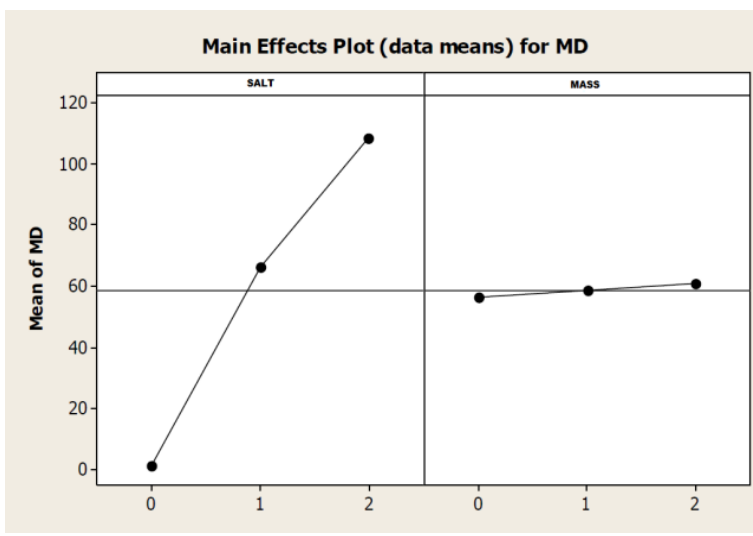

(a)

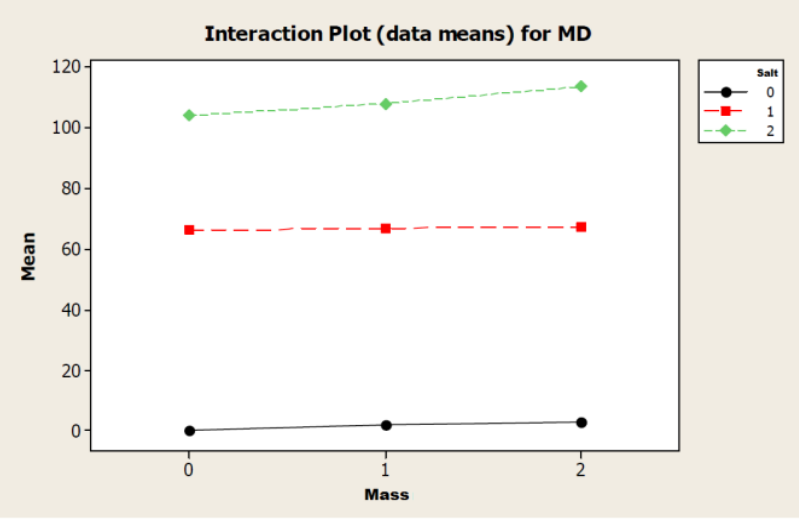

(b)

Fig. 10. Main effects of the ionic environment: (a) Isolated effects; (b) Cross effects.

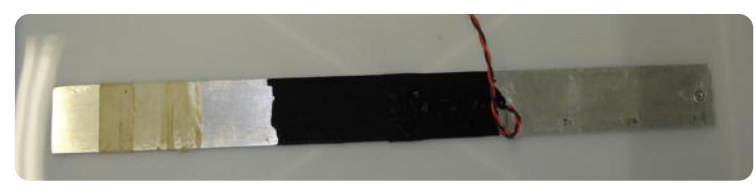

(a)

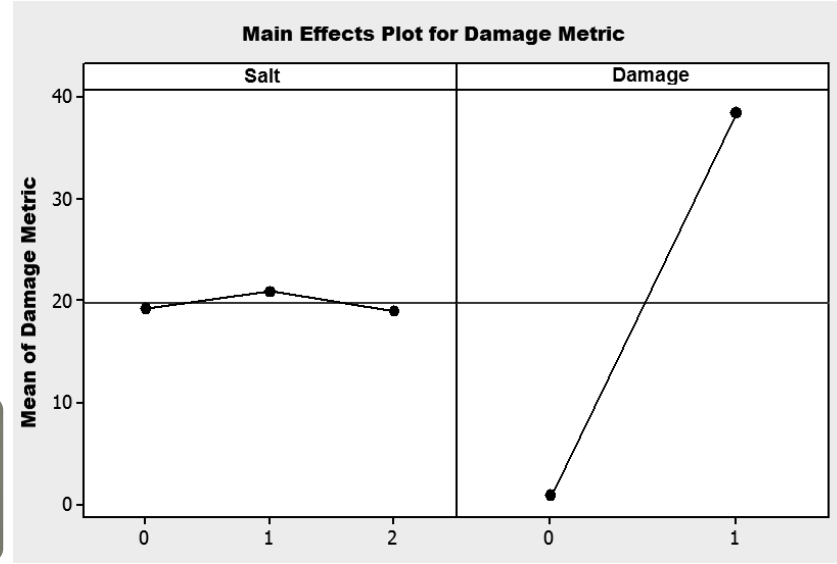

(b)

Fig. 11. (a) Shielded PZT patch; (b) Isolated effects of the ionic environment with the shielded PZT patch.

The Fig. 10a presents the main effects of the parameters of the meta-model while Fig. 10b shows the interaction effects. As can be seen in Fig. 10a, the isolated effect represented by the amount of aquarium salt is confirmed as the most significant one. However, it is worth mentioning that the amount of aquarium salt was changed from $29.2 \mathrm{~g}$ to $58.9 \mathrm{~g} \mathrm{(50 \%}$ and 100\%) and the added mass was changed only from $1 \%$ to $2 \%$ with respect to the weight of the structure. Figure 10b shows the cross effects (interaction effects) regarding the parameters of the meta-model. It can be observed that the inclination of the curves for the mass changes only slightly.

When the PZT patch is shielded (to avoid contact with the medium in which it is inserted), the effect of the ionic environment is significantly reduced, as shown in the Fig. 11.

\section{Temperature and pressure}

Moura et al. [22] first presented a study about the influence of temperature and pressure in an aluminium beam of approximately $1.0 \mathrm{~m}$ length. Both effects (temperature and pressure) were considered to better represent real conditions in which aircraft structures are submitted during normal flight operation. 
To insert damage on the surface of the beam, $\mathrm{HCl}$ (chlorhydric acid) was used to introduce a light corrosion (12 applications) at a location found 12 inches from the PZT patch, as shown in the Fig. 12a. To change the environmental conditions, a special chamber was used in such a way that temperature $\left(-60^{\circ} \mathrm{C}, 0^{\circ} \mathrm{C}\right.$, and $\left.+60^{\circ} \mathrm{C}\right)$ and pressure $1 \mathrm{~atm}$ (760 Torr - sea level), $0.23 \mathrm{~atm}(179$ Torr $-11,000 \mathrm{~m})$, and $0.01 \mathrm{~atm}(8 \mathrm{Torr}-25,500 \mathrm{~m})$ could vary. The environment conditions of the laboratory (close to $20^{\circ} \mathrm{C}$ and $1 \mathrm{~atm}$ ) were considered for the baseline signatures. Figure $12 \mathrm{~b}$ shows the experimental facility and the chamber used. To record the data, an HP4194A impedance analyzer was used and 64 averages were taken. The frequency band observed was from $40.5 \mathrm{kHz}$ to $112 \mathrm{kHz}$, with $12 \mathrm{~Hz}$ of resolution.

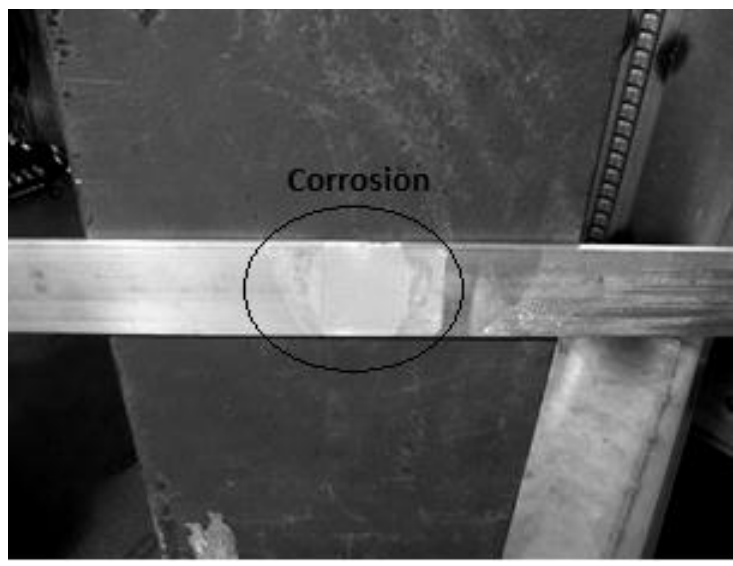

a) corrosion in the $A l$ beam

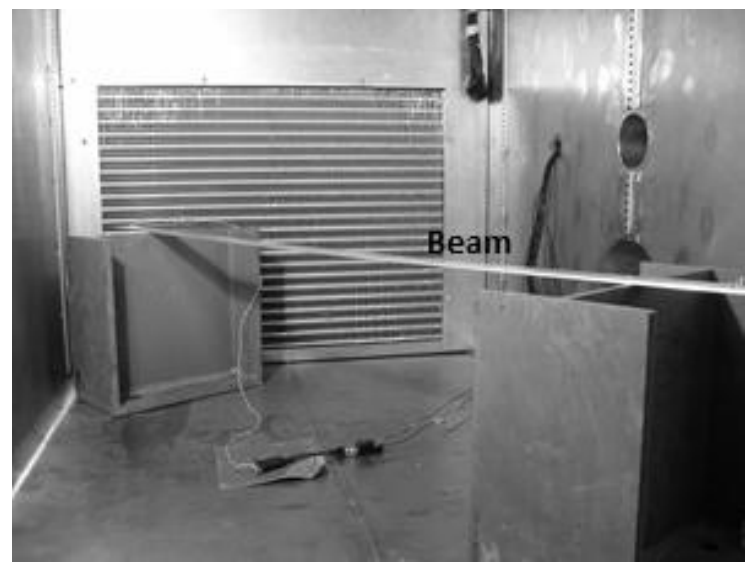

b) $A l$ beam used in this study

Fig. 12. Al beam used in the experiment (varying the pressure and the temperature).

A full factorial design $\left(3^{2} \times 2^{1}\right)$ was performed by considering the following factors: damage (two levels), temperature and pressure (three levels both) with five repetitions for each case. Both the main effects (damage, temperature and pressure) and the interaction effects (cross effects) were taken into account.

Based on the experiments made, a meta-model was built by using the software MINITAB ${ }^{\circledR}$. First, the inconsistent data were eliminated. Then, a database containing 80 cases of environmental changes was created. Then, a regression curve was obtained by using factorial design techniques, resulting the regression given by Eq. (8)

$$
\begin{aligned}
& D M=8.5651 * 10^{-3}-0.0405 * 10^{-3} D-0.0028 * 10^{-3} T-0.0001 * 10^{-3} P \\
& R-S q=74.9 \% \quad R-S q(a d j)=73.9 \%
\end{aligned}
$$

where $D$ is the adding damage, $T$ is the temperature, $P$ is the pressure and $D M$ is the resulting damage metric.

Five repetitions were performed leading to the responses \#1 to \#5. They all exhibit the same trend. The authors selected the responses \#4 and \#5 for illustration purposes. Consequently, as can be observed in Fig. 13a, b (responses \#4 and \#5), the main effects of the factors are stable and show the influence of each factor on the damage metric of the corresponding responses. From these graphics, it can be seen that when damage was inserted the damage metric was reduced. The effect of temperature exhibits an asymmetric behavior around $0^{\circ} \mathrm{C}$. However, the effect of the pressure factor is such that the corresponding damage metric increases for smaller values of pressure (i.e., for higher altitudes).

The interaction plot, Fig. 13c, shows how the interaction involving the factors can modify the damage metric of the experiment. In this case, it can be seen that the cross effect involving temperature and pressure, above $0^{\circ} \mathrm{C}$, is more significant than for lower temperatures. 


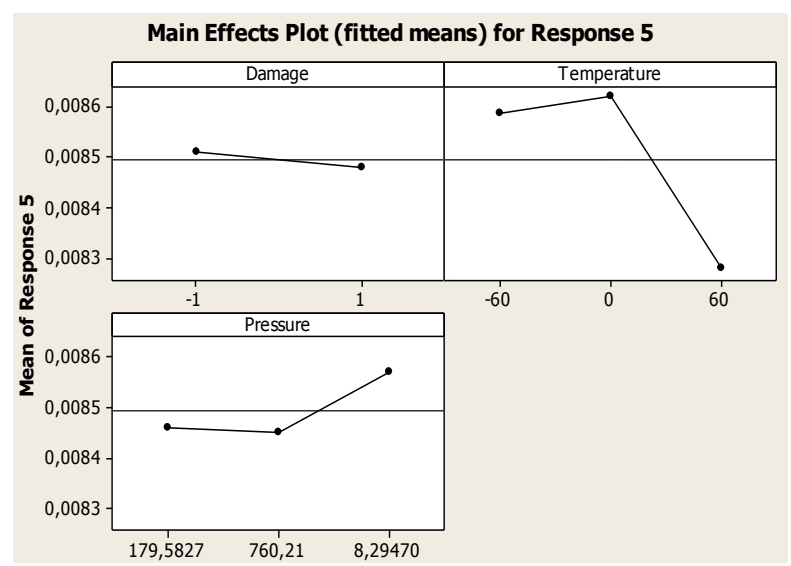

(a)

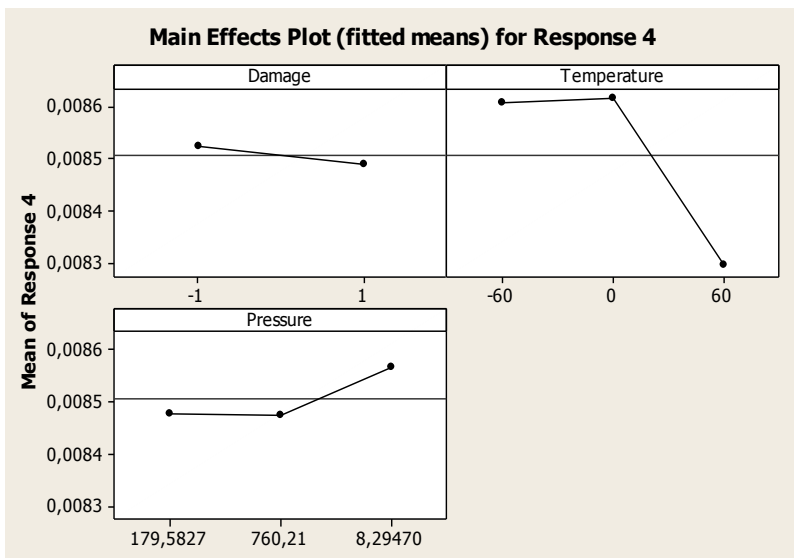

(b)

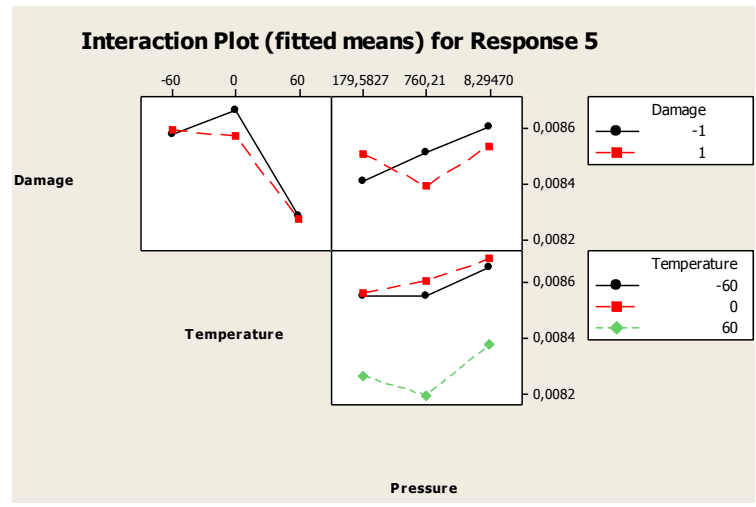

(c)

Fig. 13. Main effects of the factors (pressure and temperature): (a) Response \#5; (b) Response \#4; (c) Cross effects.

\section{Conclusion}

Regarding the influence of the shape of the piezoelectric patch it was shown that the impedance-based health monitoring technique was able to detect simulated damage both in the beam and in the plate. The meta-models show that the shape of the PZT patch does not have considerable influence in the impedance measurements in the structures tested.

The results of the effects of the electromagnetic influence on the impedance signals show that the increase of the intensity of the electromagnetic field does not affected the signals. Analyzing the regression model, a significant influence of the added mass (simulated damage) was detected in the monitoring process. However, this application should be revisited for other types of structures by studying the variation of the added mass and the variation of the intensity of the electromagnetic field.

Regarding the regression model for the ionic environment, a significant influence of the quantity of aquarium salt was detected in the monitoring process. Concerning the variables involved, i.e., the quantity of salt (unsalted, 50\% saturated, $100 \%$ saturated) and the added mass (1\% and $2 \%$ of the structure mass) to simulate damage, it is possible to note that the effect of the latter is negligible as compared to the first. This behavior seems to be confirmed by the meta-model. However, when the PZT patch is shielded, the effect of the ionic environment is significantly reduced. 
By observing the regression coefficients, it can be concluded that the effect of pressure is not important because the value of this coefficient is very low. The representation of the model by a linear meta-model is satisfactory, since the Adjusted Determination Coefficient is close to $75 \%$, meaning that $75 \%$ of the variation of damage metric can be explained by the main factors only. As expected, the influence of temperature is important and varies strongly at high temperatures. However, the same does not occur for low temperatures as found in simulated flight conditions. This means that for high temperatures it is mandatory to normalize the impedance signatures, while this may not be necessary for low temperature applications. Otherwise, false damage detection could happen due to the influence of temperature. Pressure is not of great concern in the presented technique for pressure values lower than $1.0 \mathrm{~atm}$. Further research work should consider higher values of pressure, as those found in deep water oil exploration.

\section{Acknowledgments}

The authors are grateful to FAPEMIG and CNPq, research agencies in Brazil, for funding this research work through the National Institute of Science and Technology on Smart Structures for Engineering (INCT-EIE).

\section{References}

[1] A.C. Rutherford, G. Park and C.R. Farrar, Non-linear feature identifications on self-sensing impedance measurements for structural health monitoring, Mechanical System and Signal Processing 21 (2007), 322-333.

[2] G. Park, H. Sohn, C.R. Farrar and D.J. Inman, Overview of piezoelectric impedance-based health monitoring and path forward, The Shock and Vibration Digest 35(6) (2003), 451-463.

[3] J.R.V. Moura, Jr., Fault Identification Methods for Aeronautic Structures by using meta-modeling techniques together with Impedance Methods and Smart Structures (in Portuguese), MSc Thesis, School of Mechanical Engineering, Federal University of Uberlandia, 2004.

[4] J.R.V. Moura, Jr. and V. Steffen, Jr., Impedance-based health monitoring for aeronautic structures using statistical metamodeling, Journal of Intelligent Material Systems and Structures 17 (2006), 1023-1036.

[5] C. Liang, F.P. Sun and C.A. Rogers, Coupled electromechanical analysis of adaptive material systems - Determination of the actuator power consumption and system energy transfer, Journal of Intelligent Material Systems and Structures 5 (1994), 12-20.

[6] Z. Chaudhry, T. Joseph, F. Sun and C. Rogers, Local-Area Health Monitoring of Aircraft via Piezoelectric Actuator/Sensor Patches, Smart Structures and Integrated Systems, SPIE Conference, San Diego, CA, Proceedings of the SPIE, 2443, 1995.

[7] Z. Chaudlhry, F. Lalande, A. Ganino and C. Rogers, Monitoring the Integrity of Composite Patch Structural Repair via Piezoelectric Actuators/Sensors, AIAA-1996-1074-CP, 1996.

[8] F.P. Sun, Z. Chaudhy, C. Liang and C.A. Rogers, Truss structure integrity identification using PZT sensor-actuator, Journal of Intelligent Material Systems and Structures 6 (1995), 134-139.

[9] G. Park, K. Kabeya, H.H. Cudney and D.J. Inman, Impedance- based structural health monitoring for temperature varying applications, JSME International Journal 42(2) (1999), 249-258.

[10] C.K. Soh, K. Tseng, S. Bhalla and A. Gupta, Performance of smart piezoceramic patches in health monitoring of a RC bridge, Smart Materials and Structures 9 (2000), 533-542.

[11] S. Bhalla, A.S.K. Naidu, C.W. Ong and C.K. Soh, Practical Issues in the Implementation of Electromechanical Impedance Technique for $N D E$, In Proceedings of the SPIE International Symposium on Smart Materials, Nano and Micro-Smart System, Melbourne, Australia, December 2002.

[12] V. Giurgiutiu, A.N. Zagrai, J. Bao, J. Redmond, D. Roach and K. Rackow, Active sensors for health monitoring of aging aerospace structures, International Journal of the Condition Monitoring and Diagnostic Engineering Management 6(1) (2003), 3-21.

[13] D.M. Peairs, High Frequency Modeling and Experimental Analysis for Implementation of Impedance-based Structural Health Monitoring, PhD. Thesis, Virginia Polytechnic Institute and State University, Virginia, 2006, p. 150.

[14] J.R.V. Moura, Jr., Contribution to Structural Health Monitoring Systems Applied to Aeronautic and Space Structures (in Portuguese), PhD Dissertation, Federal University of Uberlandia, 2008.

[15] H. Liu, Y. Yang and V.G.M. Annamdas, Parallel interrogation of piezoceramic transducer in electromechanical impedance technique for quick damage detection, Materials and Manufacturing Processes 5(4) (2010), 249-254.

[16] R.M. Finzi Neto, V. Steffen, Jr., D.A. Rade, C.A. Gallo and L.V. Palomino, A low-cost electromechanical impedance-based SHM architecture for multiplexed piezoceramic actuators, Structural Health Monitoring 10(4) (2011), 391-401.

[17] Y. Zhang, F. Xu, J. Chen, C. Wu and D. Wen, Electromechanical impedance response of a cracked timoshenko beam, Sensors 11 (2011), 7285-7301.

[18] V. Raju, Implementing Impedance - Based Health Monitoring, PhD. Thesis, Virginia Polytechnic Institute and State University, Virginia, 1997, p. 224.

[19] L.V. Palomino and V. Steffen, Jr., Damage Metrics Associated with electromechanical Impedance Technique for SHM Applied to a Riveted Structure, Proceedings of COBEM 2009 - 20th International Congress of Mechanical Engineering, Gramado-RS, Brazil, 2009.

[20] G.E.P. Box and N. Draper, Empirical Model Building and Response Surfaces, John Wiley \& Sons, Inc., 1986. 
[21] R.H. Myers and D.C. Montgomery, Response Surface Methodology: Process and Product in Optimization Using Designed Experiments, 1st Edition, John Wiley \& Sons, Inc., NY, USA, 1995.

[22] J.R.V. Moura, Jr., V. Steffen, Jr. and D.J. Inman, Influence of Environmental Changes in Structural Health Monitoring Analysis as Applied to Aeronautical Structures, Proc. of IMAC 2007 - Conference and Exposition on Structural Dynamics, February 2007, Orlando, FL, USA. 

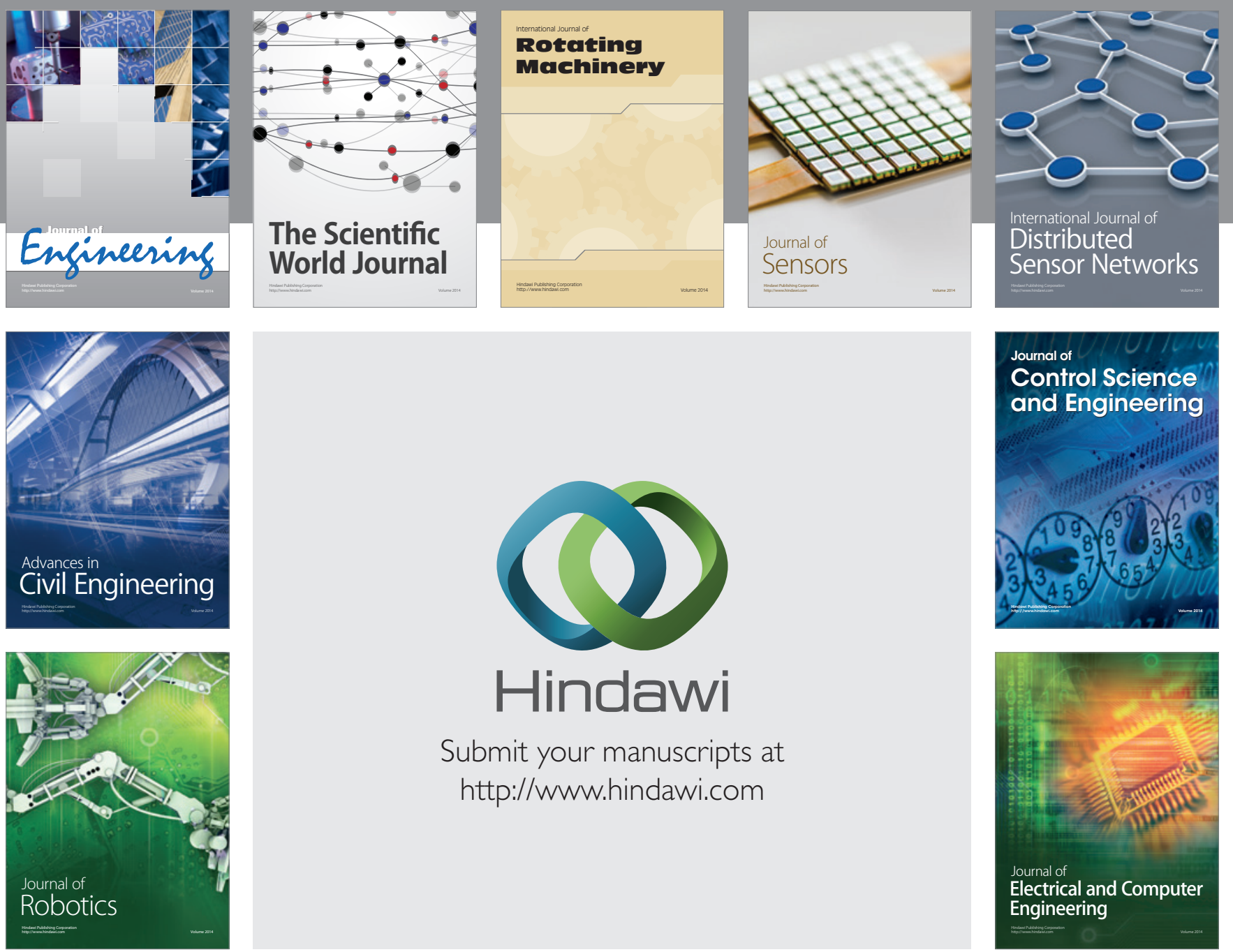

Submit your manuscripts at

http://www.hindawi.com
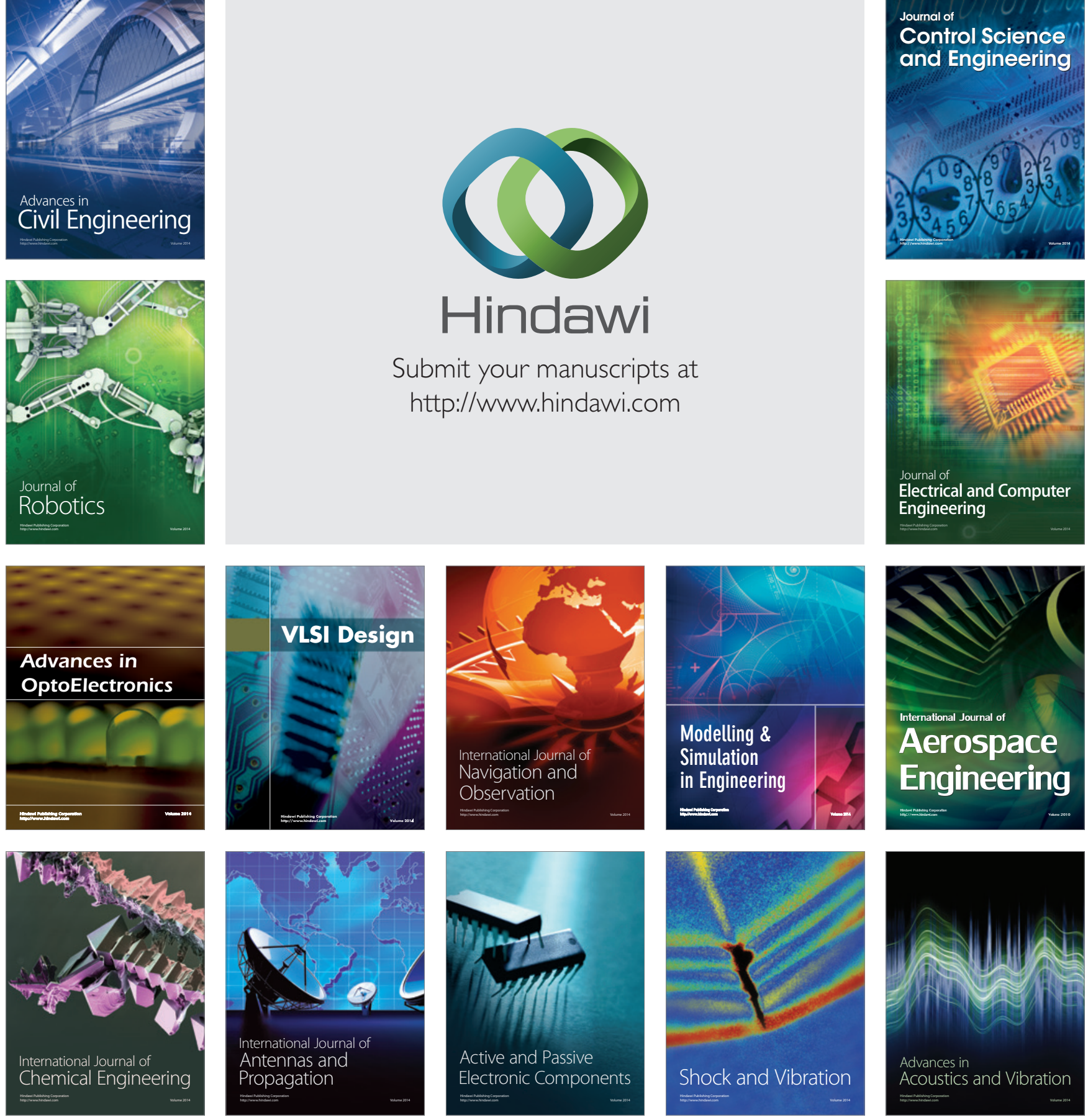